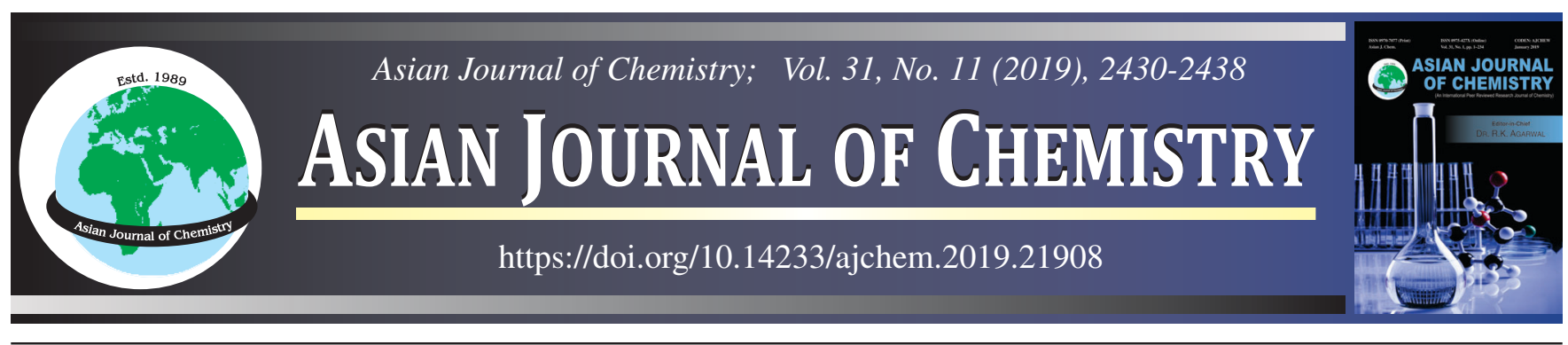

\title{
Preparation, Spectroscopic Characterization and Theoretical Studies of Transition Metal Complexes with 1-[(2-(1H-indol-3-yl)ethylimino)methyl]naphthalene-2-ol Ligand
}

\section{Vian Yamin Jirjees ${ }^{1}$, Veyan Taher Suleman $^{1}$, Abbas Ali Salih Al-Hamdani ${ }^{2, *}$ and Suzan Duraid Ahmed ${ }^{3}$}

${ }^{1}$ Department of Chemistry, College of Science, University of Dohuk, Dohuk, Iraq

${ }^{2}$ Department of Chemistry, College of Science for Women,University of Baghdad, Baghdad, Iraq

${ }^{3}$ Department of Chemistry, College of Education for Pure Science (Ibn Al-Haitham), University of Baghdad, Baghdad, Iraq

*Corresponding author: E-mail: abbas_alhamadani@yahoo.co.uk

Received: 31 December 2018;

Accepted: 21 February 2019;

Published online: 28 September 2019;

AJC-19564

| A new Schiff base [1-((2-(1H-indol-3-yl)ethylimino)methyl)naphthalene-2-ol] (HL) has been synthesized by condensing (2-hydroxy-1naphthaldehyde) with (2-(1H-indol-3-yl)ethylamine). In turn, its transition metal complexes were prepared having the general formula; $\left[\mathrm{Pt}(\mathrm{IV}) \mathrm{Cl}_{2}(\mathrm{~L})_{2}\right],\left[\operatorname{Re}(\mathrm{V}) \mathrm{Cl}_{2}(\mathrm{~L})_{2}\right] \mathrm{Cl}$ and $\left[\mathrm{Pd}(\mathrm{L})_{2}\right], 2 \mathrm{~K}\left[\mathrm{M}(\mathrm{II}) \mathrm{Cl}_{2}(\mathrm{~L})_{2}\right]$ where $\left.\mathrm{M}(\mathrm{II})=\mathrm{Co}, \mathrm{Ni}, \mathrm{Cu}\right]$ are reported. Ligand as well as metal complexes are characterized by spectroscopic techniques such as FT-IR, UV-visible, ${ }^{13} \mathrm{C} \&{ }^{1} \mathrm{H}$ NMR, mass, elemental analysis. The results | suggested that the ligand behaves like a bidentate ligand for all the synthesized complexes. On the other hand, theoretical studies of the ligand as well its metal complexes were conducted at gas phase using HyperChem 8.0. These metal complexes exhibited good antibacterial activity.

Keywords: Ethylamine, 2-Hydroxy-1-naphthaldehyde, Thermal analysis, Transition metal complexes, Schiff base.

\section{INTRODUCTION}

Schiff bases have been considered like versatile ligands which provide probability to the design metal complexes for helpful in biological activities. Numerous works based on bi-, tri- and tetradentate Schiff bases originated from different diamine or carbonyl compounds [1,2] are reported in the literature. These Schiff bases complexes containing O, N, S donor of chelates possess an unusual distribution. In these cases, presence of donor atoms at complexes could improve antitumor, antifungal, antibacterial activites, etc. [3]. Structural chemistry like symmetrical and/or unsymmetrical complexes depend upon the nature of ligands and its structural stereochemistry as well number of donor atoms also plays an important role in the enhancement of the biological activities [4]. The wide range of Schiff base complexes are applied for the organic syntheses specially in the design of medicines [5]. Moreover, they also exhibit in various biological, analytical and catalytic activities [6]. Indole based Schiff bases have extensive biological activities viz., antivirus, anticancerogenic, antibacterial, antifungal and antitumor [7]. Therefore, metal complexes of Schiff bases play a vital role at dominant gene expression, inhibition of cell division henceforth are also applied in chemotherpay, etc. However, the problem concerned for these complexes are the prepared separation in a solution that leads to highly reactive classes which are incapable into amount for drug aims like DNA. This reaction is rapid and the formation of highly interacting $\mathrm{Ni}$ (II) and $\mathrm{Pd}(\mathrm{II})$ complexes consist of bulky ligands such as triphenylphosphine [8]. In recent works, due to the presence of $d^{10}$ electronic distribution, received a lot for interest in organic, ecological and biochemistry chemistry. With respect to 20 known metal enzymes and which transition metals are in general tetrahedral coordination also associated with solid donor atoms like nitrogen [9]. Moreover, metal complexes of Schiff bases have important applications, especially in biological area in extension to their significant roles in catalysis as well as in organic composition $[7,8,10]$.

\section{EXPERIMENTAL}

All the chemicals and reagents have been acquired from the commercial sources (Sigma-Aldrich, Merck, etc.) and used without further purification.

This is an open access journal, and articles are distributed under the terms of the Attribution 4.0 International (CC BY 4.0) License. This license lets others distribute, remix, tweak, and build upon your work, even commercially, as long as they credit the author for the original creation. You must give appropriate credit, provide a link to the license, and indicate if changes were made. 
NMR spectra have been measured at DMSO- $d_{6}$ solution employing Brucker AMX $400 \mathrm{MHz}$ and JEOL Lambda 400 $\mathrm{MHz}$ spectrum. Elemental analyses $(\mathrm{C}, \mathrm{H}$ and $\mathrm{N})$ have been analyzed by using Perkin-Elmer automatic equipment model 240.B. Melting points have been measured on a Buchi SMP-20 poetical melting point device and are uncorrected. Metal contents have been analyzed using a Shimadzu 680 G Atomic Absorption Spectrometer. Conductivity measurements were conducted by employing DMSO solutions using a Jenway 4071 digital conductivity meter at room temperature. Chlorine has been specified employing the method of potentiometer titration by a 686-Titro processor-665Dosimat-Metrohm Swiss. IR analysis were carried out using Shimadzu FT-IR 3800 spectrophotometer having $\mathrm{KBr}$ or $\mathrm{CsI}$ discs in the range of $4000-400 \mathrm{~cm}^{-1}$. Electronic spectral analysis have been carried out in DMSO at $25^{\circ} \mathrm{C}$ utilizing a Shimadzu 160 Spectrophotometer. Mass spectra have been acquired through positive Electron-Influence (EI) as well Fast Atom Bombardment (FAB) has been registered at a VG auto spec micromass spectrometer.

Synthesis for ligand [1-( (2-(1H-indol-3-yl)ethylimino)methyl)naphthalene-2-ol (HL): 2-Hydroxy-1-naphthaldehyde $(0.847 \mathrm{~g}, 0.00492 \mathrm{~mol})$ dissolved in methyl alcohol solution $(10 \mathrm{~mL})$ has been mixed to an methanolic solution $(15 \mathrm{~mL})$ of 2-(1H-indol-3-yl)ethylamine $(0.788 \mathrm{~g}, 0.00492 \mathrm{~mol})$. The mixture was refluxed about $16 \mathrm{~h}$ with constant stirring. A dark yellow glossy solid has been generated and thereafter recrystallized the solid from diethyl ether:ethyl alcohol (1:1) solvent. The solid had been dehydrated over anhydrous $\mathrm{CaCl}_{2}$ (SchemeI). Yield: $89 \%$, m.p.: $247-248^{\circ} \mathrm{C}$. The ${ }^{1} \mathrm{H}$ NMR spectrum of Schiff base (HL) displays various peaks at $\delta=7.008-8.084$ $\operatorname{ppm}(\mathrm{m}, 11 \mathrm{H}, \mathrm{Ar}-\mathrm{H})$, which is attributed due to aromatic protons [11], peak at $\delta=12.117 \mathrm{ppm}(\mathrm{s}, 1 \mathrm{H}, \mathrm{N}-\mathrm{H})$ is due to the presence of proton of amine [12]. The peak at $\delta=2.103 \mathrm{ppm}$ is assigned to (tri, $\left.2 \mathrm{H}, \mathrm{C}-\mathrm{CH}_{2}\right)$ group and the peak at $\delta=3.024-3.426 \mathrm{ppm}$ (tri, $\left.2 \mathrm{H}, \mathrm{N}-\mathrm{CH}_{2}\right)[13] .{ }^{13} \mathrm{C}$ NMR (100.622 MHz, DMSO- $\left.d_{6}\right): \delta$ 177.22 (C11), 163.25 (C21), 144.67 (C14,15,16,17), 139 (C20), 127 (C5, 6), 124.31 (C13,18), 109.83 (C12), 105.95 (C7), 77.25 (C10), 56.93 (C9).

Preparation of metal complexes: The Schiff base metals complexes have been synthesized through refluxing the ethanolic solution of equimolar Schiff base and corresponding metal salts at $250 \mathrm{~mL}$ in two necked flask for $3 \mathrm{~h}$. The metal salts used in present study are chloro salts of $\operatorname{Re}(\mathrm{V}), \mathrm{Pt}(\mathrm{IV})$,
$\mathrm{Pd}(\mathrm{II}), \mathrm{Co}(\mathrm{II}), \mathrm{Ni}$ (II) and $\mathrm{Cu}(\mathrm{II})$. The solid precipitate present in the reduced volume of mother liquid has been isolated through filtration. The crude solid has been recrystallized using ethyl alcohol, dried and finally kept in vacuum desiccators.

Antibacterial activity: All the synthesized metal complexes and Schiff base ligand have been examined against $S$. aureus (Gram positive) and P. aeruginosa (Gram negative) bacteria and fungi such as Penicillium expansum, Fusarium graminearum, Macrophomina phasealina and Candida albicans, using the method of diffusion wall of agar by utilizing DMSO solvent. The condensation of compounds at this detection was $\left(10^{-3} \mathrm{M}\right)$ through employing disc sensibility check. This method includes the exposition to zone inhibition toward the diffusion from microorganism on agar plate. The plates have been brood within 24 and $48 \mathrm{~h}$ from bacteria and fungi, respectively at $37^{\circ} \mathrm{C}$.

Theoretical calculations: HyperChem 8.0 program is an advanced molecular mode, a powerful accounting package known owing to its quality, elasticity, easy to use, retractor $2 \mathrm{D}$ visualization as well as vitality for quantity chemical calculations, molecular mechanics and dynamics. In the present work, parameterization process 3 (PM3) has been applied with calculation using heat energy binding with each metal complexes. The PM3 and AMBER are more public than semiexperimental manner due to the availability for algorithms. It has parameterized primarily for organic molecules and some selected transition metals $[14,15]$.

\section{RESULTS AND DISCUSSION}

Bidentate complexes (Fig. 1) were synthesized by the interaction between metal ions and a Schiff base at molar ratio of 1:2 (metal:ligand). Synthesized solid Schiff base ligand and complexes are highly stable in the room temperature. Ligand and metals complexes are generally soluble in hot DMF and DMSO and other physico-chemical data are given in Table-1. The analytical data are satisfactorily consistent with suggested stoichiometry for complexes. It is worthwhile to mention that ligand and metal complexes can act as dyestuffs because they exhibit high molar extinction coefficients.

Mass spectrum: Mass spectrum of ligand (HL) emphasizes the possible formula by exhibiting a peak at $314.3 \mathrm{~m} / \mathrm{z}$, which is identical to that of a Schiff base moiety $\left[\left(\mathrm{C}_{21} \mathrm{H}_{18} \mathrm{~N}_{2} \mathrm{O}\right)\right.$ of atomic mass 314.38]. The chains exhibit peaks at 205, 204, 79.4 , and $36 \mathrm{~m} / \mathrm{z}$, which may be attributed to diverse fragments.

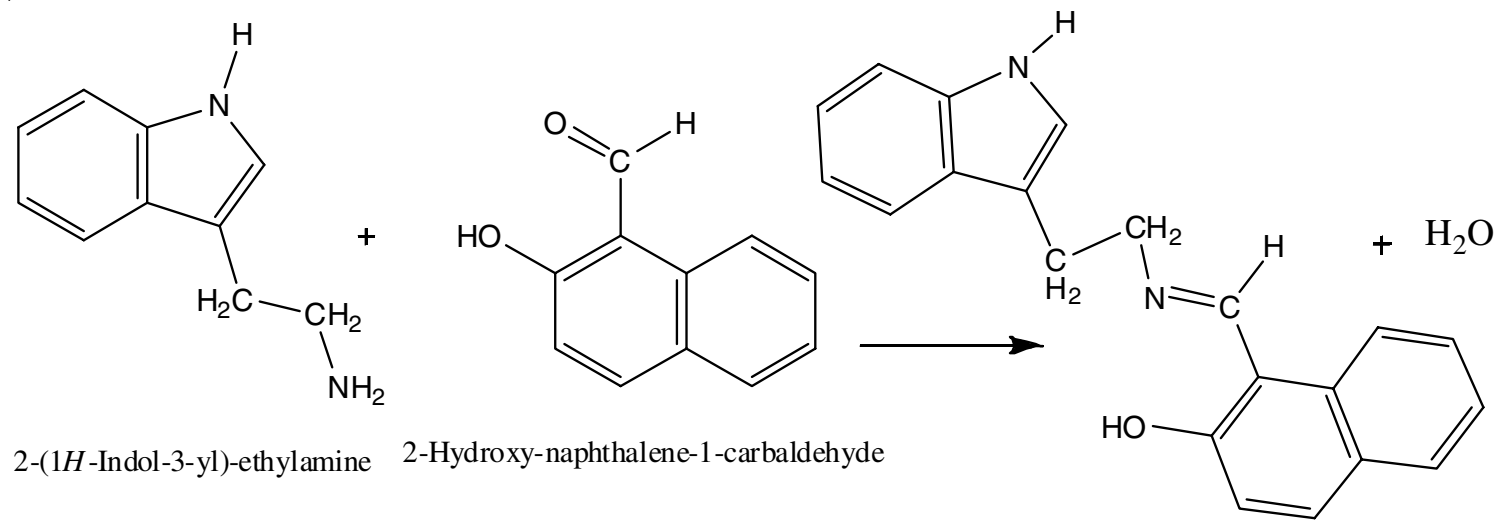

1-\{[2-(1H-Indol-3-yl)-ethylimino]-methyl $\}$-naphthalen-2-ol

Scheme-I: Synthesis route of the Schiff base 


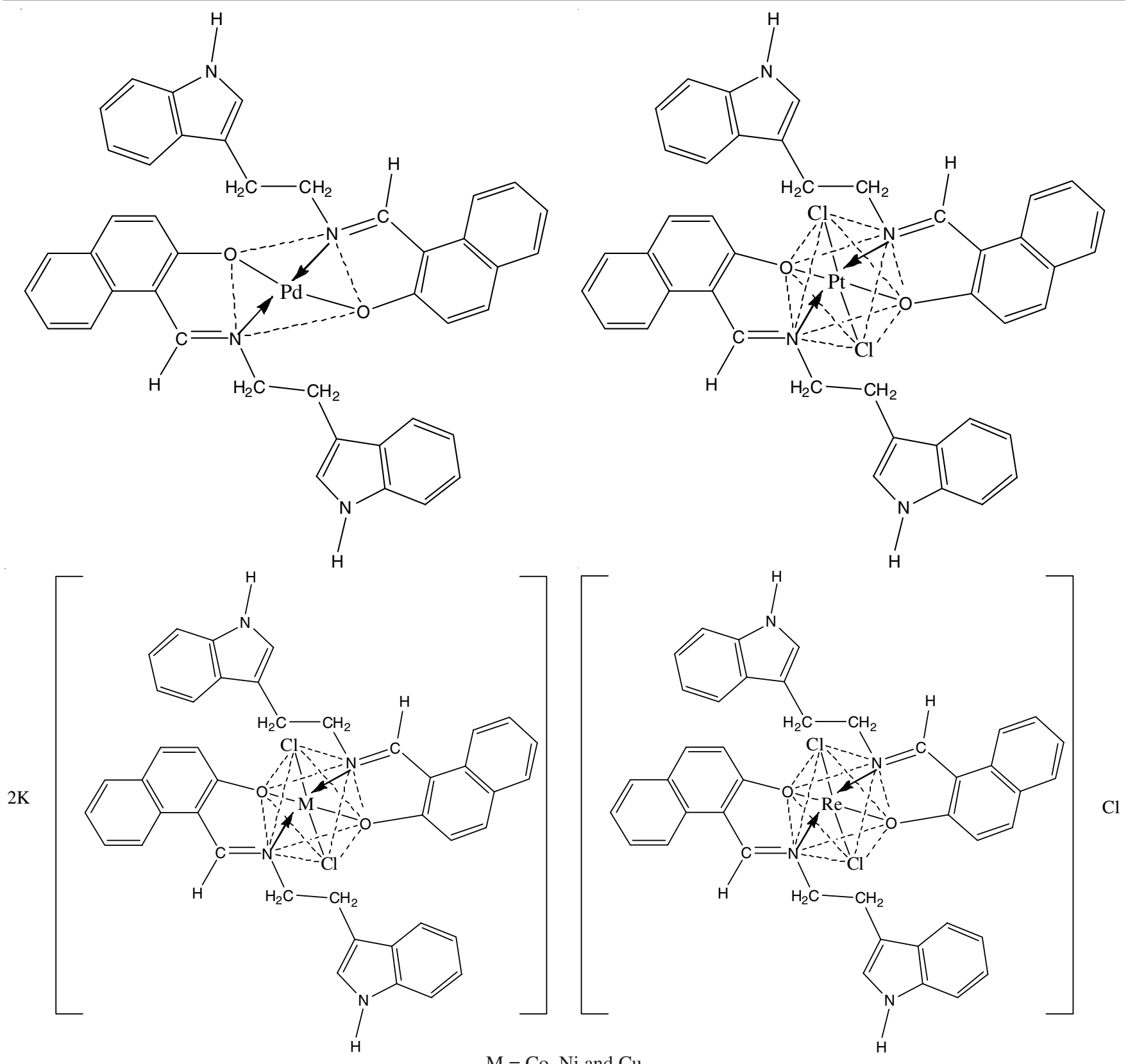

Fig. 1. Structures of the metal complexes

\begin{tabular}{|c|c|c|c|c|c|c|c|c|c|}
\hline \multirow{3}{*}{ Compounds } & \multicolumn{8}{|c|}{$\begin{array}{c}\text { TABLE-1 } \\
\text { ANALYTICAL AS WELL PHYSICAL DATA TC }\end{array}$} & \\
\hline & \multirow{2}{*}{$\begin{array}{l}\text { m.f. } \\
\text { m.w. }\end{array}$} & \multirow{2}{*}{ Colour } & \multirow{2}{*}{ m.p. $\left({ }^{\circ} \mathrm{C}\right)$} & \multirow{2}{*}{ Yield (\%) } & \multicolumn{5}{|c|}{ Elemental microanalysis (\%): Calcd. (found) } \\
\hline & & & & & $\mathrm{C}$ & $\mathrm{H}$ & $\mathrm{N}$ & M & $\mathrm{Cl}$ \\
\hline $\mathrm{HL}$ & $\begin{array}{l}\mathrm{C}_{21} \mathrm{H}_{18} \mathrm{~N}_{2} \mathrm{O} \\
314.38\end{array}$ & Dark yellow & $247-248$ & 89 & $\begin{array}{c}80.23 \\
(79.72)\end{array}$ & $\begin{array}{c}5.77 \\
(6.13)\end{array}$ & $\begin{array}{c}8.91 \\
(9.18)\end{array}$ & - & - \\
\hline$\left[\mathrm{Pd}(\mathrm{L})_{2}\right]$ & $\begin{array}{l}\mathrm{C}_{42} \mathrm{H}_{34} \mathrm{~N}_{4} \mathrm{O}_{2} \mathrm{Pd} \\
733.17\end{array}$ & Red brown & $301 \mathrm{~d}$ & 75 & $\begin{array}{c}68.80 \\
(67.91)\end{array}$ & $\begin{array}{c}4.67 \\
(5.16)\end{array}$ & $\begin{array}{c}7.64 \\
(8.07)\end{array}$ & $\begin{array}{c}14.52 \\
(13.93)\end{array}$ & - \\
\hline $\mathrm{K}_{2}\left[\mathrm{NiCl}_{2}(\mathrm{~L})_{2}\right]$ & $\begin{array}{l}\mathrm{C}_{42} \mathrm{H}_{34} \mathrm{~N}_{4} \mathrm{O}_{2} \mathrm{NiCl}_{2} \mathrm{~K}_{2} \\
834.54\end{array}$ & Dark greenish & $309 \mathrm{~d}$ & 89 & $\begin{array}{c}60.45 \\
(61.17)\end{array}$ & $\begin{array}{c}4.11 \\
(3.37)\end{array}$ & $\begin{array}{c}6.71 \\
(7.70)\end{array}$ & $\begin{array}{c}7.03 \\
(8.01)\end{array}$ & $\begin{array}{c}8.50 \\
(9.15)\end{array}$ \\
\hline$\left[\mathrm{PtCl}_{2}(\mathrm{~L})_{2}\right]$ & $\begin{array}{l}\mathrm{C}_{42} \mathrm{H}_{34} \mathrm{~N}_{4} \mathrm{O}_{2} \mathrm{Cl}_{2} \mathrm{Pt} \\
892.73\end{array}$ & Red brown & 287 & 80 & $\begin{array}{c}56.51 \\
(55.88)\end{array}$ & $\begin{array}{c}3.84 \\
(4.14)\end{array}$ & $\begin{array}{c}6.28 \\
(7.01)\end{array}$ & $\begin{array}{l}21.85 \\
(22.16)\end{array}$ & $\begin{array}{c}7.94 \\
(7.75)\end{array}$ \\
\hline $\mathrm{K}_{2}\left[\mathrm{CoCl}_{2}(\mathrm{~L})_{2}\right]$ & $\begin{array}{l}\mathrm{C}_{42} \mathrm{H}_{34} \mathrm{~N}_{4} \mathrm{O}_{2} \mathrm{CoCl}_{2} \mathrm{~K}_{2} \\
834.78\end{array}$ & Green & $327 \mathrm{~d}$ & 76 & $\begin{array}{c}60.43 \\
(61.31)\end{array}$ & $\begin{array}{c}4.11 \\
(4.49)\end{array}$ & $\begin{array}{c}6.71 \\
(7.18)\end{array}$ & $\begin{array}{c}7.06 \\
(7.13)\end{array}$ & $\begin{array}{c}8.49 \\
(9.16)\end{array}$ \\
\hline $\mathrm{K}_{2}\left[\mathrm{CuCl}_{2}(\mathrm{~L})_{2}\right]$ & $\begin{array}{l}\mathrm{C}_{42} \mathrm{H}_{34} \mathrm{~N}_{4} \mathrm{O}_{2} \mathrm{CuCl}_{2} \mathrm{~K}_{2} \\
839.39\end{array}$ & Brown & $270 \mathrm{~d}$ & 70 & $\begin{array}{c}60.10 \\
(61.01)\end{array}$ & $\begin{array}{c}4.08 \\
(5.01)\end{array}$ & $\begin{array}{c}6.67 \\
(5.89)\end{array}$ & $\begin{array}{l}7.57 \\
(8.15)\end{array}$ & $\begin{array}{c}8.45 \\
(7.78)\end{array}$ \\
\hline$\left[\mathrm{ReCl}_{2}(\mathrm{~L})_{2}\right] \mathrm{Cl}$ & $\begin{array}{l}\mathrm{C}_{42} \mathrm{H}_{34} \mathrm{~N}_{4} \mathrm{O}_{2} \mathrm{FeCl}_{3} \\
919.31\end{array}$ & Brown & $332 \mathrm{~d}$ & 86 & $\begin{array}{c}54.87 \\
(55.13)\end{array}$ & $\begin{array}{c}3.73 \\
(4.14)\end{array}$ & $\begin{array}{c}6.09 \\
(5.81)\end{array}$ & - & $\begin{array}{r}11.57 \\
(10.67)\end{array}$ \\
\hline
\end{tabular}


Pt(IV) complex: Electronic spectrum of $\left[\mathrm{PtCl}_{2}(\mathrm{~L})_{2}\right]$ confirms the possible formula because it exhibits a peak at $892 \mathrm{~m} / \mathrm{z}$, which confirms to a complex moiety $\left[\left(\mathrm{C}_{42} \mathrm{H}_{34} \mathrm{~N}_{4} \mathrm{O}_{2} \mathrm{Cl}_{2} \mathrm{Pt}\right)\right]$ of atomic mass 892.73. The peaks at 639 and $365 \mathrm{~m} / \mathrm{z}$ may be attributed to diverse fragments. Electronic spectrum of $\left[\mathrm{PdL}_{2}\right]$ indicates the possible chemical formula by exhibiting a peak at $733 \mathrm{~m} / z$, which confirms the complex moiety $\left[\left(\mathrm{C}_{42} \mathrm{H}_{34} \mathrm{~N}_{4} \mathrm{O}_{2} \mathrm{Pd}\right)\right]$ of atomic mass 733.17. Chain with peaks at 633, 562.5, 337.5, 225,205 , and $204 \mathrm{~m} / \mathrm{z}$ may be attributed to diverse fragments [16].

Electronic spectra and magnetic moments studies: Table-2 indicates the values of ligand absorption and intensive absorption is observed at 33090 and $39087 \mathrm{~cm}^{-1}$, which may be assigned to $\pi \rightarrow \pi^{*}$ also $n \rightarrow \pi^{*}$, respectively. The value of magnetic moment 3.70 B.M. indicates that Co(II) complex has octahedral geometry $[17,18]$. The electronic spectrum of $\mathrm{Co}$ (II) complex appeared as two wide peaks at 16266.1, 14749.2 and $11001.1 \mathrm{~cm}^{-1}$, which were designated as ${ }^{4} \mathrm{~T}_{1} \mathrm{~g} \rightarrow{ }^{4} \mathrm{~T}_{1} \mathrm{~g}(\mathrm{P})$ $\left(v_{3}\right),{ }^{4} \mathrm{~T}_{1 \mathrm{~g}} \rightarrow{ }^{4} \mathrm{~A}_{2 \mathrm{~g}}\left(\mathrm{~V}_{2}\right)$ and ${ }^{4} \mathrm{~T}_{1 \mathrm{~g}} \rightarrow{ }^{4} \mathrm{~T}_{\mathrm{gg}}(\mathrm{F})\left(\mathrm{V}_{1}\right)$ transition, respectively. Furthermore, the peak at $24937.6 \mathrm{~cm}^{-1}$, designated as $\mathrm{M} \rightarrow \mathrm{L}$ (C.T.) and $37174.7 \mathrm{~cm}^{-1}$, was assigned to a ligand field. The Ni(II) complex shows peaks at 23041, 20576 and 12722.6 $\mathrm{cm}^{-1}$, which indicated ${ }^{3} \mathrm{~A}_{2 \mathrm{~g}} \rightarrow{ }^{3} \mathrm{~T}_{1 \mathrm{~g}}(\mathrm{P}),{ }^{3} \mathrm{~A}_{2 \mathrm{~g}} \rightarrow{ }^{3} \mathrm{~T}_{1 \mathrm{~g}}(\mathrm{~F})$ and ${ }^{3} \mathrm{~A}_{2 \mathrm{~g}}$ $\rightarrow{ }^{3} \mathrm{~T}_{2 \mathrm{~g}}$ (F) transition, respectively, and at $36363.6 \mathrm{~cm}^{-1}$, which was attributed to a ligand field proposing octahedral geometry of ligands around $\mathrm{Ni}(\mathrm{II})$ ions. The value of magnetic moment (2.91 B.M.) of $\mathrm{Ni}$ (II) complex is consistent with octahedral geometry $[13,17,18]$. The Pt(IV) complex jointly with the $\mu_{\text {eff }}$ value (Table-3) shows four peaks in 24390.2, 20618.5 and $19230.7 \mathrm{~cm}^{-1}$ whom specified into ${ }^{1} \mathrm{~A}_{1 \mathrm{~g}} \rightarrow{ }^{1} \mathrm{~T}_{3 \mathrm{~g}},{ }^{1} \mathrm{~A}_{1 \mathrm{~g}} \rightarrow{ }^{1} \mathrm{~T}_{2 \mathrm{~g}}$ also ${ }^{1} \mathrm{~A}_{\mathrm{lg}} \rightarrow{ }^{1} \mathrm{~T}_{1 \mathrm{~g}}$ transition, respectively as well $32786.8 \mathrm{~cm}^{-1}$ assigned to $\mathrm{M} \rightarrow \mathrm{L}$ (C.T.) also $37174.7 \mathrm{~cm}^{-1}$, assigned to ligand field, which suggested octahedral geometry of $\mathrm{Pt}(\mathrm{IV})$ complex $[17,19]$. In case of $\mathrm{Pd}(\mathrm{II})$ complex, bands were assigned to the ligand field, ${ }^{1} \mathrm{~A}_{1 \mathrm{~g}} \rightarrow{ }^{1} \mathrm{~B}_{1 \mathrm{~g}}$ and ${ }^{1} \mathrm{~A}_{1 \mathrm{~g}} \rightarrow{ }^{1} \mathrm{~A}_{2 \mathrm{~g}}$ (35971.2, 20283.9, and 13071.8$) \mathrm{cm}^{-1}$, respectively; furthermore, this indicates square planar geometry around the $\mathrm{Pd}(\mathrm{II})$ ion, moreover, the value of magnetic moment to this complex is consistent with square planar geometry $[17,20]$. Electronic spectra for $\mathrm{Cu}$ (II) complexes showed low energy absorption bands at 20242.9 and 36363.6 $\mathrm{cm}^{-1}$, which can be attributed to the transitions ${ }^{2} \mathrm{E}_{\mathrm{g}} \rightarrow{ }^{2} \mathrm{~T}_{2 \mathrm{~g}}$ and ligand field, respectively. These bands indicate the octahedral geometry of $\mathrm{Cu}$ (II) ion and also the magnetic moment value of 1.66 B.M. of $\mathrm{Cu}(\mathrm{II})$ complex is also consistent with octahedral

TABLE-2

ELECTRONIC DATA, MAGNETIC MOMENTS AND MOLAR CONDUCTIVITY OF THE METAL COMPLEXES

\begin{tabular}{|c|c|c|c|c|c|c|c|c|}
\hline Complexes & ABS & $\lambda(\mathrm{nm})$ & $v\left(\mathrm{~cm}^{-1}\right)$ & $\varepsilon\left(\mathrm{L} \mathrm{mol}^{-1} \mathrm{~cm}^{-1}\right)$ & Assignment & $\begin{array}{c}\Omega\left(\mathrm{Ohm}^{-1}\right. \\
\left.\mathrm{cm}^{2} \mathrm{~mol}^{-1}\right)\end{array}$ & $\mu_{\text {eff }}$ (B.M) & $\begin{array}{l}\text { Proposed } \\
\text { geometry }\end{array}$ \\
\hline \multirow{4}{*}[\mathrm{ReCl}_{2}(\mathrm{L})_{2}]{$\mathrm{Cl}$} & 2.459 & 275 & 36363.6 & 2459 & Ligand field + C.T. & \multirow{4}{*}{82} & \multirow{4}{*}{2.95} & \multirow{4}{*}{ Octahedra } \\
\hline & 0.983 & 489 & 20449.8 & 983 & ${ }^{3} \mathrm{~T}_{1 \mathrm{~g}} \rightarrow{ }^{3} \mathrm{~A}_{2 \mathrm{~g}}$ & & & \\
\hline & 0.546 & 637 & 15698.5 & 546 & ${ }^{3} \mathrm{~T}_{1 \mathrm{~g}} \rightarrow{ }^{3} \mathrm{~T}_{1 \mathrm{~g}(\mathrm{P})}$ & & & \\
\hline & 0.208 & 715 & 13986 & 208 & ${ }^{3} \mathrm{~T}_{1 \mathrm{~g}} \rightarrow{ }^{3} \mathrm{~T}_{2 \mathrm{~g}(\mathrm{~F})}$ & & & \\
\hline \multirow{5}{*}[\mathrm{PtCl}_{2}(\mathrm{L})_{2}]{} & 1.688 & 269 & 37174.7 & 16880 & Ligand field & \multirow{5}{*}{25} & \multirow{5}{*}{ Diamagnetic } & \multirow{5}{*}{ Octahedral } \\
\hline & 2.109 & 305 & 32786.8 & 21090 & C.T. & & & \\
\hline & 0.741 & 410 & 24390.2 & 741 & ${ }^{1} \mathrm{~A}_{1 \mathrm{~g}} \rightarrow{ }^{1} \mathrm{~T}_{3 \mathrm{~g}}$ & & & \\
\hline & 0.627 & 485 & 20618.5 & 627 & ${ }^{1} \mathrm{~A}_{1 \mathrm{~g}} \rightarrow{ }^{1} \mathrm{~T}_{2 \mathrm{~g}}$ & & & \\
\hline & 0.588 & 520 & 19230.7 & 588 & ${ }^{1} \mathrm{~A}_{\mathrm{lg}} \rightarrow{ }^{1} \mathrm{~T}_{1 \mathrm{~g}}$ & & & \\
\hline \multirow{3}{*}[\mathrm{Pd}(\mathrm{L})_{2}]{} & 2.414 & 278 & 35971.2 & 24140 & Ligand field & \multirow{3}{*}{27} & \multirow{3}{*}{ Diamagnetic } & \multirow{3}{*}{$\begin{array}{l}\text { Square } \\
\text { planar }\end{array}$} \\
\hline & 0.605 & 493 & 20283.9 & 605 & ${ }^{1} \mathrm{~A}_{1 \mathrm{~g}} \rightarrow{ }^{1} \mathrm{~B}_{1 \mathrm{~g}}$ & & & \\
\hline & 0.101 & 765 & 13071.8 & 101 & ${ }^{1} \mathrm{~A}_{1 \mathrm{~g}} \rightarrow{ }^{1} \mathrm{~A}_{2 \mathrm{~g}}$ & & & \\
\hline \multirow{2}{*}{$\mathrm{K}_{2}\left[\mathrm{CuCl}_{2}(\mathrm{~L})_{2}\right]$} & 2.082 & 275 & 36363.6 & 20820 & Ligand Field & \multirow{2}{*}{145} & \multirow{2}{*}{1.66} & \multirow{2}{*}{ Octahedral } \\
\hline & 0.604 & 494 & 20242.9 & 604 & ${ }^{2} \mathrm{E}_{\mathrm{g}} \rightarrow{ }^{2} \mathrm{~T}_{2 \mathrm{~g}}$ & & & \\
\hline \multirow{5}{*}{$\mathrm{K}_{2}\left[\mathrm{CoCl}_{2}(\mathrm{~L})_{2}\right]$} & 1.850 & 269 & 37174.7 & 1850 & Ligand Field & \multirow{5}{*}{159} & \multirow{5}{*}{3.70} & \multirow{5}{*}{ Octahedral } \\
\hline & 2.472 & 401 & 24937.6 & 401 & C.T. & & & \\
\hline & 0.061 & 615 & 16266.1 & 615 & ${ }^{4} \mathrm{~T}_{1 \mathrm{~g}} \rightarrow{ }^{4} \mathrm{~T}_{1 \mathrm{~g}(\mathrm{P})}$ & & & \\
\hline & 0.106 & 678 & 14749.2 & 678 & ${ }^{4} \mathrm{~T}_{1 \mathrm{~g}} \rightarrow{ }^{4} \mathrm{~A}_{2 \mathrm{~g}(\mathrm{~F})}$ & & & \\
\hline & 0.009 & 909 & 11001.1 & 909 & ${ }^{4} \mathrm{~T}_{1 \mathrm{~g}} \rightarrow{ }^{3} \mathrm{~T}_{\mathrm{lg}(\mathrm{F})}$ & & & \\
\hline \multirow{4}{*}{$\mathrm{K}_{2}\left[\mathrm{NiCl}_{2}(\mathrm{~L})_{2}\right]$} & 2.401 & 275 & 36363.6 & 24010 & Ligand Field & \multirow{4}{*}{166} & \multirow{4}{*}{2.91} & \multirow{4}{*}{ Octahedral } \\
\hline & 0.203 & 434 & 23041 & 203 & ${ }^{3} \mathrm{~A}_{2 \mathrm{~g}} \rightarrow{ }^{3} \mathrm{~T}_{1 \mathrm{~g}(\mathrm{P})}$ & & & \\
\hline & 0.232 & 486 & 20576 & 232 & ${ }^{3} \mathrm{~A}_{2 \mathrm{~g}} \rightarrow{ }^{3} \mathrm{~T}_{1 \mathrm{~g}(\mathrm{~F})}$ & & & \\
\hline & 0.084 & 786 & 12722.6 & 84 & ${ }^{3} \mathrm{~A}_{2 \mathrm{~g}} \rightarrow{ }^{3} \mathrm{~T}_{2 \mathrm{~g}(\mathrm{~F})}$ & & & \\
\hline
\end{tabular}

TABLE-3

FTIR SPECTRUM DATA $\left(\mathrm{cm}^{-1}\right)$ OF THE METAL COMPLEXES

\begin{tabular}{|c|c|c|c|c|c|c|}
\hline Compounds & $\mathrm{C}-\mathrm{H}$ aromatic aliphatic & $v(\mathrm{~N}-\mathrm{H})$ & $v(\mathrm{C}=\mathrm{N})$ & $v(\mathrm{O}-\mathrm{H})$ & $v(\mathrm{M}-\mathrm{N})$ & $v(\mathrm{M}-\mathrm{O})$ \\
\hline$\overline{\mathrm{HL}}$ & 3021,2987 & 3243 & 1694 & 3575 & - & - \\
\hline$\left[\mathrm{ReCl}_{2}(\mathrm{~L})_{2}\right] \mathrm{Cl}$ & 3026,2982 & 3240 & 1666 & - & 534 & 484,423 \\
\hline$\left[\mathrm{PtCl}_{2}(\mathrm{~L})_{2}\right]$ & 3043,2988 & 3237 & 1635 & - & 586,536 & 491,474 \\
\hline$\left[\mathrm{Pd}(\mathrm{L})_{2}\right]$ & 3022,2979 & 3239 & 1676 & - & 530 & 480,424 \\
\hline $\mathrm{K}_{2}\left[\mathrm{CuCl}_{2}(\mathrm{~L})_{2}\right]$ & 3043,2957 & 3241 & 1634 & - & 582,536 & 500,474 \\
\hline $\mathrm{K}_{2}\left[\mathrm{CoCl}_{2}(\mathrm{~L})_{2}\right]$ & 3023,2937 & 3240 & 1682 & - & 534 & 484,423 \\
\hline $\mathrm{K}_{2}\left[\mathrm{NiCl}_{2}(\mathrm{~L})_{2}\right]$ & 3021,2968 & 3236 & 1637 & - & 580,540 & 490,420 \\
\hline
\end{tabular}


geometry $[17,19]$. The spectrum for $\operatorname{Re}(\mathrm{V})$ complex, $\left(\mu_{\text {eff }} 2.95\right)$ exhibits four peaks at 36363.6, 20449.8, 15698.5 and 13986 $\mathrm{cm}^{-1}$, which indicated ligand field (C.T.), ${ }^{3} \mathrm{~T}_{1 \mathrm{~g}} \rightarrow{ }^{3} \mathrm{~A}_{2 \mathrm{~g}},{ }^{3} \mathrm{~T}_{1 \mathrm{~g}} \rightarrow{ }^{3} \mathrm{~T}_{1 \mathrm{~g}}$ (p) and ${ }^{3} \mathrm{~T}_{1 \mathrm{~g}} \rightarrow{ }^{3} \mathrm{~T}_{2 \mathrm{~g}}$ (F) transition, respectively. Furthermore, these data indicated the octahedral geometry for $\operatorname{Re}(\mathrm{V})$ complex $[17,19]$. Moreover, molar conductivity of these complexes are also found consistent for electrolytes of every complex with 1:2 electrolyte and 1:1 electrolytes for Re complex but with non-electrolytes for $\mathrm{Pt}$ and $\mathrm{Pd}$ complexes.

Infrared analysis: The ligand exhibits the characteristic bands at 3575, 1307, 3243 and $1694 \mathrm{~cm}^{-1}$ which is attributed to $v(\mathrm{OH})$ phenolic, $v(\mathrm{C}-\mathrm{O})$ phenolic, $v(\mathrm{~N}-\mathrm{H})$ and $v(\mathrm{C}=\mathrm{N})$ azomethine, respectively. The IR spectra of the complexes exhibited that ligand bands shifts due to the formation of complexes (Table-3) $[21,22]$. Furthermore, the $v(C=N)$ band of ligand was observed at $1694 \mathrm{~cm}^{-1}$ and also this band shifted to lower frequencies through $\left(28-22 \mathrm{~cm}^{-1}\right)$ in complexes. This indicates that the ligand was coordinated to the metal ions through $\mathrm{N}$ atom (azomethine) [11,13].

The results also revealed that the ligand was coordinated with metal ions by $\mathrm{N}$-atom. Reduction in bond order on complexation, may be caused by delocalization of electron metal intensity ( $\mathrm{t}_{2 \mathrm{~g}}$ ) into the $\pi$-system of ligand. These shifts indicate the coordination of the ligand through nitrogen from the group of azomethine into metal ions. The $v(\mathrm{~N}-\mathrm{H})$ band of free ligand at $3243 \mathrm{~cm}^{-1}$ did not shift in the complexes. At low frequencies, the complexes exhibited bands near 582-534 $\mathrm{cm}^{-1}$, which are attributed to $v(\mathrm{M}-\mathrm{N})$. Additionally, bands near $403-378 \mathrm{~cm}^{-1}$ were assigned to $\mathrm{v}(\mathrm{M}-\mathrm{Cl})$ with complexes. Therefore, the $\mathrm{Cl}$ atom was considered coordinated with all complexes, except for palladium complex, which did not exhibit a band to the $v(\mathrm{M}-\mathrm{Cl})[11,23,24]$. Moreover, ligand offers a wide band on approximately $3575 \mathrm{~cm}^{-1}$ because intramolecular $\mathrm{H}$-bonded of hydroxide group. This band vanishes at their corresponding metal complexes showing the coordination of phenol oxygen into metal ion by removing the proton [13]. Comparison of experimental results and theoretical vibration frequencies are listed in Table-4.

Theoretical study: Vibrational spectra of the ligand and complexes were calculated by using a semi-empirical (PM3) method. The distortion may occurred because the approximate harmonic oscillators lack an electron connection. Previous study [23] reported that frequencies coupled for Hartre-Fock Theory proximite a quantum harmonic oscillator rounding which tends to be $10 \%$ high. Theoretically possible structures from metal complexes with ligand have been calculated to match the most possible pattern building steady structure. These forms display the calculated optimal geometries with ligands and its metal complexes. The results from PM3 method from calculation at gas phase for the binding energies and heat values of formation of $\mathrm{Co}(\mathrm{II}), \mathrm{Ni}(\mathrm{II}), \mathrm{Pd}(\mathrm{II}), \mathrm{Re}(\mathrm{V})$ and $\mathrm{Pt}(\mathrm{IV})$ complexes are listed in Table-5.

Electrostatic potential: Electron allocation decides the electrostatic potential (EP) of molecules and characterizes the interaction of energy to the molecular system for positive dot transport. Thus, it is useful in finding sites from reaction at a molecular positive charged types whom tend into offensive molecule wherever EP is robustly negative electrophilic link [25]. Electrostatic potential from free ligands has been calculated and used as a 3D contour to examine the reactive locations of molecules, their stereochemistry, and calculate reaction rates of numerous reactive species, including soft electrophiles and nuclophiles in terms of characteristics from border orbitals (HOMO and LUMO). The interference between HOMO and LUMO values has been plotted in a manner similar to $3 \mathrm{D}$ contour to obtain more information on these molecules. The results

TABLE-4

COMPARISON FROM EXPERIMENTAL AND THEORETICAL VIBRATION FREQUENCIES $\left(\mathrm{cm}^{-1}\right)$ WITH LIGAND AS WELL METAL COMPLEXES

\begin{tabular}{|c|c|c|c|c|c|c|c|}
\hline Compounds & $v(\mathrm{OH})$ & $\begin{array}{c}\mathrm{v}(\mathrm{CH}) \\
\text { aromatic }\end{array}$ & $v(\mathrm{CH})$ aliph. & $v(\mathrm{C}=\mathrm{N})$ & $v(\mathrm{~N}-\mathrm{H})$ & $v(\mathrm{M}-\mathrm{N})$ & $v(\mathrm{M}-\mathrm{O})$ \\
\hline \multirow{3}{*}{$\mathrm{HL}$} & $3575^{*}$ & $3021 *$ & $2987 *$ & 1694* & $3243 *$ & \multirow{3}{*}{-} & \multirow{3}{*}{-} \\
\hline & $3434 * *$ & $3122 * *$ & $2344 * *$ & $1756 * *$ & $3432 * *$ & & \\
\hline & $0.039 * * *$ & $-0.033 * * *$ & $0.215 * * *$ & $-0.036 * * *$ & $-0.058 * * *$ & & \\
\hline \multirow{3}{*}[\mathrm{ReCl}_{2}(\mathrm{L})_{2}]{$\mathrm{Cl}$} & \multirow{3}{*}{-} & $3026^{*}$ & $2982 *$ & $1666^{*}$ & $3240 *$ & $534^{*}$ & $484 *, 423 *$ \\
\hline & & $3121 * *$ & $3011 * *$ & $1755^{* *}$ & $3222 * *$ & $555 * *$ & $400 * *$ \\
\hline & & $-0.031 * * *$ & $-0.006 * * *$ & $-0.053 * * *$ & $0.005 * * *$ & $-0.039 * * *$ & $0.173 * * *$ \\
\hline \multirow{3}{*}[\mathrm{PtCl}_{2}(\mathrm{L})_{2}]{} & \multirow{3}{*}{-} & $3043 *$ & $2988^{*}$ & $1635^{*}$ & $3237 *$ & $586^{*}, 536^{*}$ & $491^{*}, 474^{*}$ \\
\hline & & $3001 * *$ & $2900 * *$ & $1600 * *$ & $3333 * *$ & $566 * *$ & $444 * *$ \\
\hline & & $0.013 * * *$ & $0.029 * * *$ & $0.021 * * *$ & $-0.029 * * *$ & $0.034 * * *$ & $0.095 * * *$ \\
\hline \multirow{3}{*}[\operatorname{Pd}(\mathrm{L})_{2}]{} & \multirow{3}{*}{-} & $3022 *$ & $2979 *$ & $1676^{*}$ & $3239 *$ & $530^{*}$ & $480 *, 424 *$ \\
\hline & & $3222 * *$ & $2900 * *$ & $1600 * *$ & $3222 * *$ & $500 * *$ & $422 * *$ \\
\hline & & $-0.066 * * *$ & $0.026 * * *$ & $0.045^{* * *}$ & $0.005 * * *$ & $0.056^{* * *}$ & $0.120 * * *$ \\
\hline \multirow{3}{*}{$\mathrm{K}_{2}\left[\mathrm{CuCl}_{2}(\mathrm{~L})_{2}\right]$} & \multirow{3}{*}{-} & $3043 *$ & $2957 *$ & $1634^{*}$ & $3241 *$ & $582 *, 536 *$ & $500^{*}, 474^{*}$ \\
\hline & & $3000 * *$ & $2900 * *$ & $1633^{* *}$ & $3222 * *$ & $555 * *$ & $444 * *$ \\
\hline & & $0.014 * * *$ & $0.019 * * *$ & $0.0006 * * *$ & $0.005 * * *$ & $0.046 * * *$ & $0.112 * * *$ \\
\hline \multirow{3}{*}{$\mathrm{K}_{2}\left[\mathrm{CoCl}_{2}(\mathrm{~L})_{2}\right]$} & \multirow{3}{*}{-} & $3023 *$ & $2937^{*}$ & $1682 *$ & $3240 *$ & $534 *$ & $484^{*}, 423 *$ \\
\hline & & $3000 * *$ & $2922 * *$ & $1650 * *$ & $3400 * *$ & $500 * *$ & $488 * *$ \\
\hline & & $0.007 * * *$ & $0.005 * * *$ & $0.019 * * *$ & $-0.049 * * *$ & $0.063 * * *$ & $-0.008 * * *$ \\
\hline \multirow{3}{*}{$\mathrm{K}_{2}\left[\mathrm{NiCl}_{2}(\mathrm{~L})_{2}\right]$} & \multirow{3}{*}{-} & $3021 *$ & $2968 *$ & $1637 *$ & $3236 *$ & $580^{*}, 540^{*}$ & $490^{*}, 420^{*}$ \\
\hline & & $3000 * *$ & $2933 * *$ & $1600 * *$ & $3255^{* *}$ & $530 * *$ & $488 * *$ \\
\hline & & $0.006 * * *$ & $0.011 * * *$ & $0.022 * * *$ & $-0.005 * * *$ & $0.086^{* * *}$ & $0.004 * * *$ \\
\hline
\end{tabular}

*Empirical frequency. **Theoretical frequency. $* * *$ Error $\%$ because of the main difference in empirical mensuration as well as theoretical treatments from spectrum vibration. 

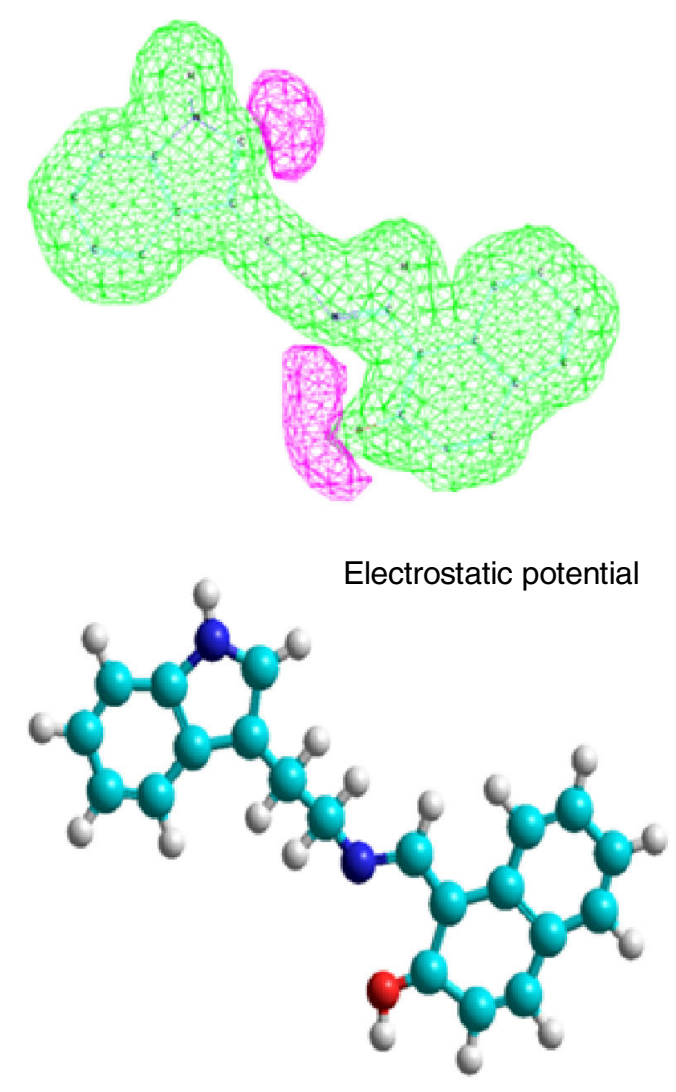

HL
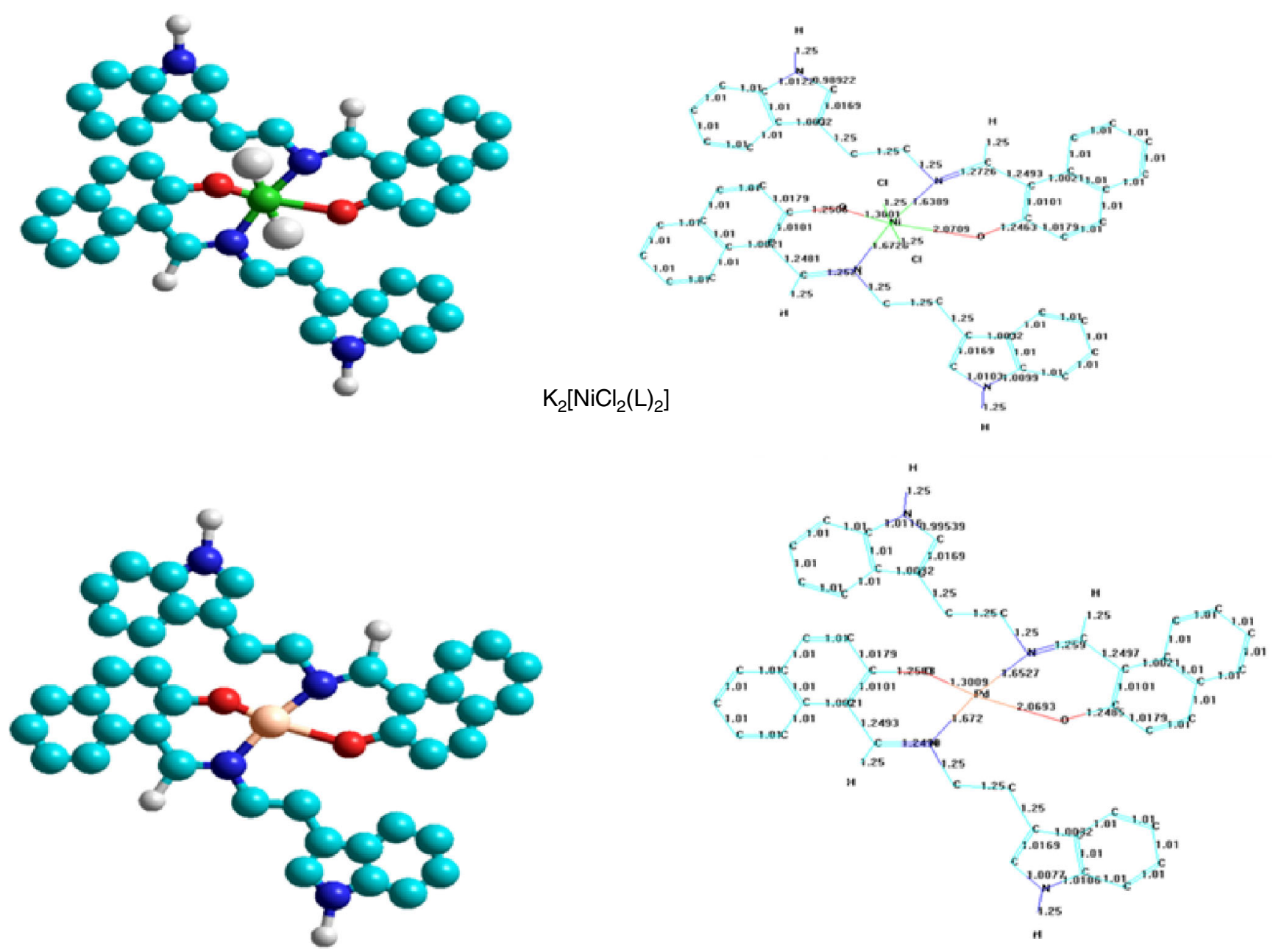

$\left.\operatorname{Pd}(L)_{2}\right]$ 

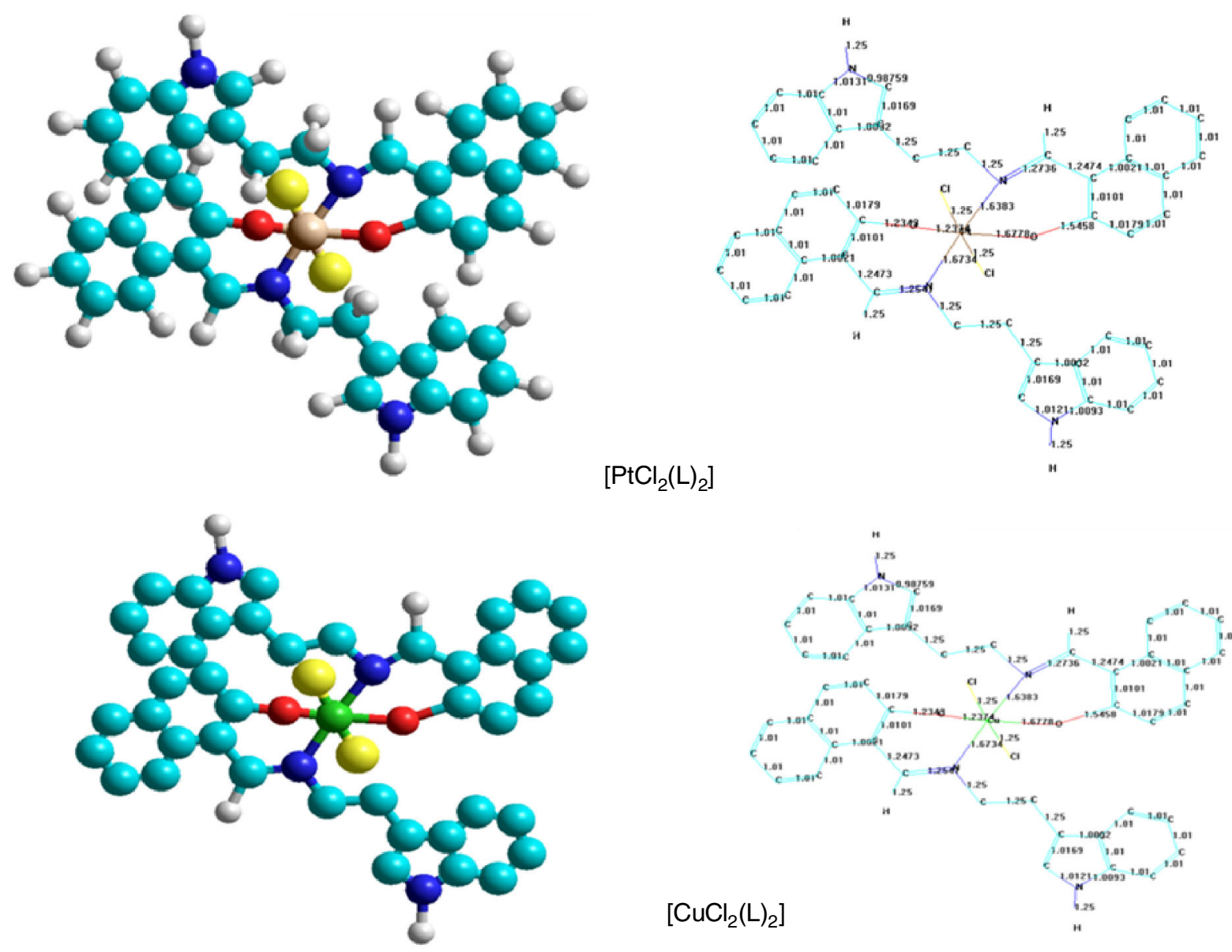

$\left[\mathrm{CuCl}_{2}(\mathrm{~L})_{2}\right]$
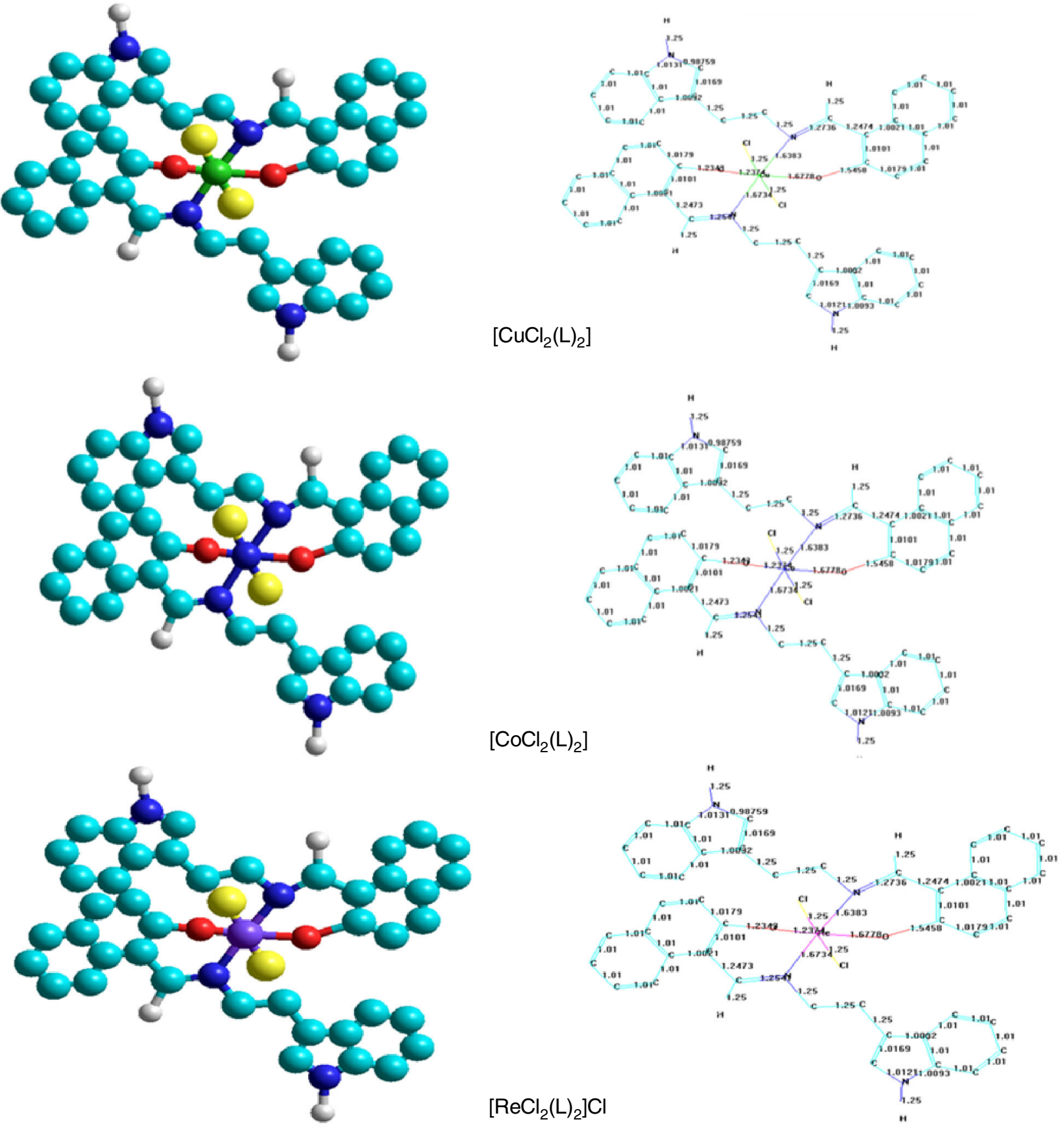

Fig. 2. Conformational structures of ligand and its metal complexes 
TABLE-5

CONFORMATION ENERGETIC $\left(\mathrm{KJ} \mathrm{mol}^{-1}\right)$ ALSO DIPOLE MOMENT WITH LIGAND ALSO METAL COMPLEXES

\begin{tabular}{lrrrr}
\hline \multicolumn{1}{c}{ Compounds } & Binding energy & Heat of fashioning & Electronic energy & Dipole moment (debye) \\
\hline $\mathrm{HL}$ & -3237.3109465 & 741.1420535 & -451373.8437952 & 3.257 \\
{$\left[\mathrm{ReCl}_{2}(\mathrm{~L})_{2}\right] \mathrm{Cl}$} & -1137.3109465 & -1137.3109465 & -322137.3109465 & 1.257 \\
{$\left[\mathrm{PtCl}_{2}(\mathrm{~L})_{2}\right]$} & -937.3109465 & -937.3109465 & -113237.3109465 & 2.570 \\
{$\left[\mathrm{Pd}(\mathrm{L})_{2}\right]$} & -837.3109465 & -837.3109465 & -493237.3109465 & 2.700 \\
$\mathrm{~K}_{2}\left[\mathrm{CuCl}_{2}(\mathrm{~L})_{2}\right]$ & -37.3109465 & -7.3109465 & 3.2570000 & 1.200 \\
$\mathrm{~K}_{2}\left[\mathrm{CoCl}_{2}(\mathrm{~L})_{2}\right]$ & -67.3109465 & -3.3109465 & 3.2570000 & 2.200 \\
$\mathrm{~K}_{2}\left[\mathrm{NiCl}_{2}(\mathrm{~L})_{2}\right]$ & -68.3109465 & 13.3109465 & 3.2570000 & 0.900 \\
\hline
\end{tabular}

TABLE-6

ANTIBACTERIAL EFFICACY DATA OF LIGAND AND ITS METAL COMPLEXES AS INHIBITION ZONE (mm)

\begin{tabular}{|c|c|c|c|c|c|c|c|c|c|c|c|c|c|c|c|c|}
\hline \multirow{3}{*}{ Compounds } & \multicolumn{8}{|c|}{ Bacteria } & \multicolumn{8}{|c|}{ Fungi } \\
\hline & \multicolumn{2}{|c|}{$\begin{array}{c}\text { Stphyococcus } \\
\text { aureus }\end{array}$} & \multicolumn{2}{|c|}{$\begin{array}{l}\text { Bacillus } \\
\text { subtlis }\end{array}$} & \multicolumn{2}{|c|}{$\begin{array}{c}\text { Pseudomonas } \\
\text { aeruginosa }\end{array}$} & \multicolumn{2}{|c|}{$\begin{array}{c}\text { Escherichia } \\
\text { coli }\end{array}$} & \multicolumn{2}{|c|}{$\begin{array}{c}\text { Penicillium } \\
\text { expansum }\end{array}$} & \multicolumn{2}{|c|}{$\begin{array}{c}\text { Fusarium } \\
\text { graminearum }\end{array}$} & \multicolumn{2}{|c|}{$\begin{array}{c}\text { Macrophomina } \\
\text { phascolina }\end{array}$} & \multicolumn{2}{|c|}{$\begin{array}{l}\text { Candida } \\
\text { albicans }\end{array}$} \\
\hline & $\mathrm{I}$ & II & $\mathrm{I}$ & II & $\mathrm{I}$ & II & $\mathrm{I}$ & II & $\mathrm{I}$ & II & $\mathrm{I}$ & II & $\mathrm{I}$ & II & $\mathrm{I}$ & II \\
\hline $\mathrm{HL}$ & 12 & - & 20 & 18 & 12 & - & 24 & 12 & 46 & 36 & - & - & - & - & +++ & +++ \\
\hline $\mathrm{ReCl}_{5}$ & 20 & 10 & 13 & - & 15 & 12 & 8 & - & +++ & 56 & - & - & - & - & +++ & +++ \\
\hline $\mathrm{H}_{2} \mathrm{PtCl}_{6} \cdot 6 \mathrm{H}_{2} \mathrm{O}$ & 26 & 15 & - & - & 12 & - & - & - & 38 & 28 & 38 & 33 & - & - & - & - \\
\hline $\mathrm{PdCl}_{2}$ & 12 & 10 & - & - & 15 & - & - & - & - & - & - & - & - & - & 20 & 10 \\
\hline $\mathrm{CuCl}_{2} \cdot 2 \mathrm{H}_{2} \mathrm{O}$ & 15 & 12 & 23 & 25 & 23 & 18 & 8 & - & - & - & - & - & - & - & - & - \\
\hline $\mathrm{CoCl}_{2} \cdot 6 \mathrm{H}_{2} \mathrm{O}$ & 40 & 25 & 20 & 12 & 16 & 15 & 8 & 12 & - & - & - & - & - & - & - & - \\
\hline $\mathrm{NiCl}_{2} \cdot 6 \mathrm{H}_{2} \mathrm{O}$ & 30 & 12 & 19 & 24 & 20 & 14 & 24 & 30 & - & - & - & - & - & - & - & - \\
\hline$\left[\mathrm{ReCl}_{2}(\mathrm{~L})_{2}\right] \mathrm{Cl}$ & 24 & - & 23 & 19 & 24 & 18 & 30 & 12 & 12 & - & 12 & - & 24 & 20 & 12 & 8 \\
\hline$\left[\mathrm{PtCl}_{2}(\mathrm{~L})_{2}\right]$ & 18 & - & 23 & 19 & 22 & 15 & 40 & 32 & 23 & 19 & 24 & 14 & 25 & 20 & 23 & 15 \\
\hline$\left[\mathrm{Pd}(\mathrm{L})_{2}\right]$ & 30 & 25 & 25 & 20 & 28 & 18 & - & 28 & 23 & 19 & 25 & 20 & - & - & 8 & - \\
\hline $\mathrm{K}_{2}\left[\mathrm{CuCl}_{2}(\mathrm{~L})_{2}\right]$ & 28 & 22 & 12 & 21 & 12 & - & - & - & 30 & 28 & 30 & 22 & 30 & 20 & 25 & 17 \\
\hline $\mathrm{K}_{2}\left[\mathrm{CoCl}_{2}(\mathrm{~L})_{2}\right]$ & 24 & 20 & 32 & 13 & 22 & 15 & 15 & 22 & 23 & 17 & 19 & 12 & - & - & 29 & 18 \\
\hline $\mathrm{K}_{2}\left[\mathrm{NiCl}_{2}(\mathrm{~L})_{2}\right]$ & 38 & 30 & 21 & 18 & 28 & 18 & 19 & 8 & 23 & 20 & - & - & 25 & 14 & - & - \\
\hline Control & 0 & 0 & 0 & 0 & 0 & 0 & 0 & 0 & 0 & 0 & 0 & 0 & 0 & 0 & 0 & 0 \\
\hline
\end{tabular}

(I): High concentration; (II): Low concentration; (+++): Strong inhibition

revealed that LUMO from transition metal ion prefers to react for HOMO from nitrogen atoms of azo-Schiff base ligand (Fig. 2).

Microbiological activities: The isolated bacteria were inoculated in tubes for nutrient broth (NB). The classified NB $\left(1 \mathrm{~cm}^{3}\right)$ was homogenized and heated using burners at $45^{\circ} \mathrm{C}$. Homogeneous commentary have been casted to petri plates. Discs of filter paper (dia. $4 \mathrm{~mm}$ ) were introduced on the discs. After the solidified form of NB cooled and a formed layer of $2 \times 10^{-5} \mathrm{dm}^{3}$ of the test compounds was applied. After nursery for $24 \mathrm{~h}$ in thermostat at $25-27^{\circ} \mathrm{C}$, inhibition (sterilized) zone diameters (involving disc) have been measured in $\mathrm{mm}$. An inhibition of diameter zone of $>7 \mathrm{~mm}$ indicated that the tested compound is effective against bacteria [26]. Antibacterial activity of the synthesized ligand, metal salts and complexes have been screened against Gram-negative bacteria such as Escherichia coli and Pseudomonas aeruginosa and Gram-positive bacteria such as Bacillus subtlis and Staphylococcus aureus.

All the metal complexes, metal salts and ligand have been tested for their activity against fungi such as Penicillium expansum, Fusarium graminearum, Macrophomina phasealina and Candida albicans. The results are shown in Table-6.

\section{Conclusion}

All compounds were prepared and characterized using spectroscopic techniques such as FT-IR, UV-visible, ${ }^{13} \mathrm{C} \&{ }^{1} \mathrm{H}$ NMR, mass and elemental analysis. Theoretical studies of the ligand and its complexes have also been performed using the quantum chemical calculation asymptotic through density func- tional theory. A good correlation between empirical and experimental results were found. The prepared ligand and its metal complexes have been examined for their anti-bacterial and antifungal activities.

\section{CONFLICT OF INTEREST}

The authors declare that there is no conflict of interests regarding the publication of this article.

\section{REFERENCES}

1. G. Bandoli, M. Nicolini, U. Mazzi and F.J. Refosco, J. Chem. Soc., Dalton Trans., 11, 2505 (1984); https://doi.org/10.1039/DT9840002505.

2. K.J.C. van Bommel, W. Verboom, H. Kooijman, A.L. Spek and D.N. Reinhoudt, Inorg. Chem., 37, 4197 (1998); https://doi.org/10.1021/ic980125t.

3. K.C. Gupta and A.K. Sutar, Coord. Chem. Rev., 252, 1420 (2008); https://doi.org/10.1016/j.ccr.2007.09.005.

4. P. Mayer, K.C. Potgieter and T.I.A. Gerber, Polyhedron, 29, 1423 (2010); https://doi.org/10.1016/j.poly.2010.01.013.

5. G.A. Lane, W.E. Geiger and N.G. Connelly, J. Am. Chem. Soc., 109, 402 (1987); https://doi.org/10.1021/ja00236a018.

6. Y.G. Li, D.H. Shi, H.L. Zhu, H. Yan and S.W. Ng, Inorg. Chim. Acta, 360, 2881 (2007);

https://doi.org/10.1016/j.ica.2007.02.019.

7. S. Güveli and B. Ülküseven, Polyhedron, 30, 1385 (2011); https://doi.org/10.1016/j.poly.2011.02.041.

8. S. Priyarega, P. Kalaivani, R. Prabhakaran, T. Hashimoto, A. Endo and K. Natarajan, J. Mol. Struct., 1002, 58 (2011); https://doi.org/10.1016/j.molstruc.2011.06.046. 
9. J.J. Fernandez, A. Fernandez, D. Vázquez-Garcia, M. López-Torres, A. Suárez, N. Gómez-Blanco and J.M. Vila, Eur. J. Inorg. Chem., 2007, 5408 (2007); https://doi.org/10.1002/ejic.200700478.

10. W. Al-Zoubi, A.A.S. Al-Hamdani and Y.G. Ko, Sep. Sci. Technol., 52, 1052 (2017); https://doi.org/10.1080/01496395.2016.1267756.

11. W.A. Zoubi, A.A.S. Al-Hamdani and Y.G. Ko, J. Chil. Chem. Soc., 60 , 2774 (2015); https://doi.org/10.4067/S0717-97072015000100003.

12. R.M. Sliverstien and X.F. Webser, Spctrometric Identification of Organic Compounds, John Wiley \& Sons, Inc.: USA, edn 7 (2005).

13. W. Al Zoubi, A.A.S. Al-Hamdani, S.D. Ahmed and Y.G. Ko, Appl. Organomet. Chem., 32, e3895 (2018); https://doi.org/10.1002/aoc.3895.

14. D.B. Cook, Handbook of Computational Quantum Chemistry, Oxford University Press: New York, p. 149 (1998).

15. A.A.S. Al-Hamdani1, N.Kh.G. Al-Dulyme, S.D. Ahmed and H.M. Basheer, J. Al-Nahrain Univ. Sci., 20, 49 (2017)

16. A.A.S. Al Hamdani and W. Al Zoubi, Spectrochim. Acta A Mol. Biomol. Spectrosc., 137, 75 (2015);

https://doi.org/10.1016/j.saa.2014.07.057.
17. A.B.P. Lever, Inorganic Electronic Spectroscopy, Elsevier Publishing Company: Amsterdam, London, edn 6, p. 121 (1968).

18. S.A. Shaker, H.A. Mohammed and A.A.S. Al-Hamdani, Aus. J. Basic. App. Sci., 4, 5178 (2010).

19. W. Al Zoubi, A.A.S. AlHamdani, I.P. Widiantara, R.G. Hamoodah and Y.G. Ko, J. Phys. Org. Chem., 30, 3707 (2017); https://doi.org/10.1002/poc.3707.

20. A. Al-Hamdani, S. Ahmed, S. Shake and Z. Hasan, J. Baghdad Sci., 13, 105 (2016); https://doi.org/10.21123/bsj.2016.13.2.2NCC.00105.

21. K. Nakamoto, Infrared and Raman Spectra of Inorganic and Coordination Compounds, Wiley-Inter Science: New York (1997).

22. R.M. Silverstein, G.C. Bassler and T.C. Movril, Spectroscopic Identification of Organic Compounds, Wiley: New York, edn 4 (1981).

23. A.A.S. Al-Hamdani and G.H. Rehab, J. Baghdad Sci., 13, 770 (2016); https://doi.org/10.21123/bsj.13.4.770-781.

24. W. Al Zoubi, A.A.S. Al-Hamdani, S.D. Ahmed and Y.G. Ko, J. Phys. Org. Chem., 31, e3752 (2018); https://doi.org/10.1002/poc.3752.

25. A.A.S. Al-Hamdani and Sh.A. Shaker, Orient. J. Chem., 27, 825 (2011).

26. J.M. Andrews, J. Antimicrob. Chemother, 56, 60 (2005); https://doi.org/10.1093/jac/dki124. 